\title{
CORTICAL REGIONS CONNECTED WITH AUTONOMIC CARDIOVASCULAR REGULATION
}

\author{
Darina Georgieva-Hristova ${ }^{1}$, Silva Andonova ${ }^{1}$, Evgeniya Kalevska ${ }^{1}$, Veselinka Nestorova ${ }^{2}$, \\ Ivan Dzedzev², Diana Radkova ${ }^{3}$ \\ ${ }^{1}$ Department of Neurology and Neuroscience, Medical University of Varna, Bulgaria, \\ ${ }^{2}$ Department of professional disease, Medical University of Varna, Bulgaria, \\ ${ }^{3}$ Department of Infectious, Epidemiology and Tropical medicine, \\ Medical University of Varna, Bulgaria,
}

\begin{abstract}
Violations of the autonomic nervous system are common in patients with cerebrovascular diseases and adversely affect the quality of life. The central autonomic network combines multiple reciprocally connected cortical, subcortical and stem structures. The insular cortex is involved in visceral motor and sensory regulation, including that of the cardiovascular system. Brain lesions including insula and other prefrontal cortical areas are particularly important in provoking arrhythmia. Stroke involving insular cortex is associated with poor prognosis. The right insular cortex plays a dominant role in modulating sympathetic tone and the left insular cortex in parasympathetic tone, which clinically correlates with more frequent arrhythmias and arrhythmic death after a stroke affecting the insula in the right hemisphere. The insular cortex is located in the middle cerebral artery and its structure is exposed to a high risk of injury.
\end{abstract}

Keywords: cortex insularis, cerebrovascular disease, arrhythmia, arterial hypertension, cardiac dysfunction

Bulgaria is one of the first places in the world in morbidity and mortality from cerebrovascular disease. According to the National Statistical Institute, cerebrovascular mortality for 2013 was 281.7 per 100 000 people, the leading mortality from malignant neoplasms. Frequent and significant social consequences of stroke are movement disorders, but disorders of the autonomic nervous system are also com-

Address for correspondence:

Darina Georgieva

Department of Neurology,

Medical University of Varna

55 Marin Drinov Str.

9002 Varna

e-mail:darina_georgieva@abv.bg

Received: October 24, 2014

Accepted: March 11, 2015 mon in patients with cerebrovascular diseases and adversely affect the quality of life.

The central autonomic network combines multiple reciprocally connected cortical, subcortical and stem structures (1). Neurocardiological classical literature focuses mainly on subcortical regions of the central autonomic nervous system, namely the activation of different receptors located mainly in the heart and blood vessels. The collected information is integrated into the region of the brain stem and, through mechanisms of negative feedback, information is transferred to the target organ of the peripheral autonomic nervous system.

In 1980 Hachinski et al. reported an association between cortical lesions affected by stroke and cardiovascular dysregulation and prove that the cardiovascular system is regulated by cortical modulation. 
Darina Georgieva-Hristova, Silva Andonova, Evgeniya Kalevska et al.

Modern neuroimaging data including positron emission tomography and magnetic resonance imaging, showed that cortex insularis, amygdala, hypothalamus, periaqueductal gray matter, nucleus parabrachialis, nucleus tractus solitarius and medulla ventrolateralis are an integral part of the internal regulatory system by which the brain controls visceromotor, neuroendocrine and pulmonary systems as well as pain sensitation (1).

Cortex insularis has reciprocal connections with the gyrus cingularis frontalis, amygdala, cortex entorhinalis and cortex temporalis orbitofrontal field and has afferent connections with formation hippocampalis (3).

\section{THE ROLE OF THE INSULAR COR- TEX IN THE AXIS: BRAIN-HEART}

Experimental and human studies suggest that brain lesions including insula and other prefrontal cortical areas are particularly important in provoking arrhythmia. Cortex insularis is involved in visceral motor and sensory regulation, including that of the cardiovascular system (4), and also plays an important role in the limbic-autonomic integration and participates in the perception of emotional significance. Interhemispheric correlation between both insulas offers an integrated modeling of the cardiovascular system and diencephalic and brain-stem vegetative centers (4). This indicates that communication between the two insulas is required for balanced interhemisferic cardiovascular control.

Stroke involving cortex insularis is associated with poor prognosis. Arrhythmogenic structures are: the amygdala, hippocampus, before the department of gyrus cingularis, hypothalamus, nucleus parabrachialis. Stimulation of these areas causes significant sympathetic activation, which is resistant even after vagal stimulation.

Experimental studies suggest that the two brain hemispheres have different effect on the nature and the severity of arrhythmias after stroke. Pulse is increased by inactivation of the left hemisphere and decreases in deactivation of the right hemisphere. While a person's right island crust plays a dominant role in modulating the sympathetic tone, the left cortex insularis-in the parasympathetic tone. Sided lesions increased sympathetic tone, but also decreased parasympathetic tone. All this clinically correlat- ed with more frequent arrhythmias and arrhythmic death after a stroke affecting the insula in the right hemisphere (3).

Specifically, insular cortex lesion on the right is associated with atrial fibrillation, atrioventricular block, premature contractions and inversion of T-wave. Strokes in the right hemisphere relate with supraventricular tachycardia, and in the left hemisphere-with chamber arrhythmias (3).

When compared to the lesion of the left insular peel or damage to regions beyond the insular cortex lesion in the right, the insular cortex was associated with an increased risk of death (3).

Furthermore, when there was an effect on the insula, higher levels of serum epinephrine, elevated blood pressure and longer QT intervals were observed. In addition, female sex and hypokalemia are independent risk factors for QT prolongation, which subsequently leads to malignant arrhythmias and poor prognosis (2).

The insula has a viscerotropic organization. In 1990 Oppenheimer and Cechetto confirm the cardiac chronotropic organization of the insula posterior (5). In particular, the locations producing tachycardia are located in superior posterior insula; places associated with the production of bradycardia are in inferior posterior insula. Reduced activation of the insula frontalis may reflect, as well as suppress, the parasympathetic heart tone.

Acute cerebral diseases also affect the cardiovascular mechanisms autonomous control of blood pressure. Systolic and diastolic blood pressure are transiently increased immediately after a stroke, but are governed spontaneously after 7 days. The degree of the increase in blood pressure vary depending on the type of stroke and is greater after lacunar infarctions and haemorrhagic strokes (3).

\section{CONCLUSION}

Cortex insularis is located in the middle cerebral artery and its structure tends to be at a high risk of cerebrovascular disease. ECG changes, reduced HRV and poor prognosis of stroke occurred more often in patients with stroke involving the right insular area. 


\section{REFERENCES}

1. Benarroch $\mathrm{E}$. The central autonomic network: Functional organization, dysfunction and perspective. Mayo Clinic Proc, 1993;68:988-1001.

2. Katsanos AH, Korantzopoulos P, Tsivgoulis G, Kyritsis AP, Kosmidou M, Giannopoulos S. Electrocardiographic abnormalities and cardiac arrhythmias in structural brain lesions. 2013;167(2):328-34.

3. Korpelainen JT, Sotaniemi KA, Myllylä VV, Cheung RTF, Hachinski VC.Autonomic nervous system disorders in stroke 1999. The insula and cerebrogenic sudden death. Arch Neurol, 2000;57:1685-1688.

4. Lane RD, McRae K, Reiman EM, Chen K, Ahern GL,Thayer JF. Neural correlates of heart rate variability. Neuroimage, 2009;44:213-22.

5. Oppenheimer SM and Cechetto DF. Cardiac chronotropic organization of the rat insular cortex.

Brain Res, 1990;533,66-72. 